\title{
Injuries of the Conus Medullaris
}

\author{
T. K. F. Taylor, ${ }^{1}$ M. J. R. Coolican ${ }^{2}$
}

${ }^{1}$ Department of Orthopaedic and Traumatic Surgery, Spinal Injuries Unit, The Royal North Shore Hospital, Sydney, St Leonards, NSW 2065, Australia. ${ }^{2}$ University of Sydney, NSW, Australia.

\section{Summary}

Eighteen patients with traumatic lesions of the conus medullaris were reviewed with particular reference to bowel, bladder and sexual functions. The averge follow-up was 4 years. The injury most frequently occurred with a burst fracture of the first lumbar vertebra. All patients could walk on discharge but 4 required some form of external support. Regular bladder habit was regained in all patients but none had normal bladder sensation. Urinary tract infections were quite uncommon and there was good correlation between the incidence of infections and the degree of bladder control. No patient had developed chronic renal failure or chronic urinary tract infection. Bowel control was similarly preserved but at a somewhat lower level of competence. Eleven of 16 males had been able to undertake intercourse though with variable adequacy. Potency was linked with the degree of preservation of genital sensation. The conclusion is that the prognosis for conus lesions is somewhat better than is widely appreciated.

Key words: Spinal cord Injuries; Conus medullaris; Prognosis.

The conus medullaris is the finely tapered distal end of the spinal cord which in the adult terminates at, or about, the upper border of the second lumbar vertebra. The disability resulting from a conus injury relates to the functions served by the sacral outflow (S2, 3 and 4 ). While the anatomy in this region is variable, the conus is always located within one vertebral level of the $\mathrm{L} 1 / 2$ interspace. It contains both the sacral and coccygeal spinal segments. With fractures of the first lumbar vertebral body the structure is at risk and may sustain irreversible damage. Whilst the surrounding mobile nerve roots of more proximal cord origin remain relatively unharmed: the concept of root escape, pure conus lesions is decidedly uncommon.

The present study was performed to determine the long term prognosis on 


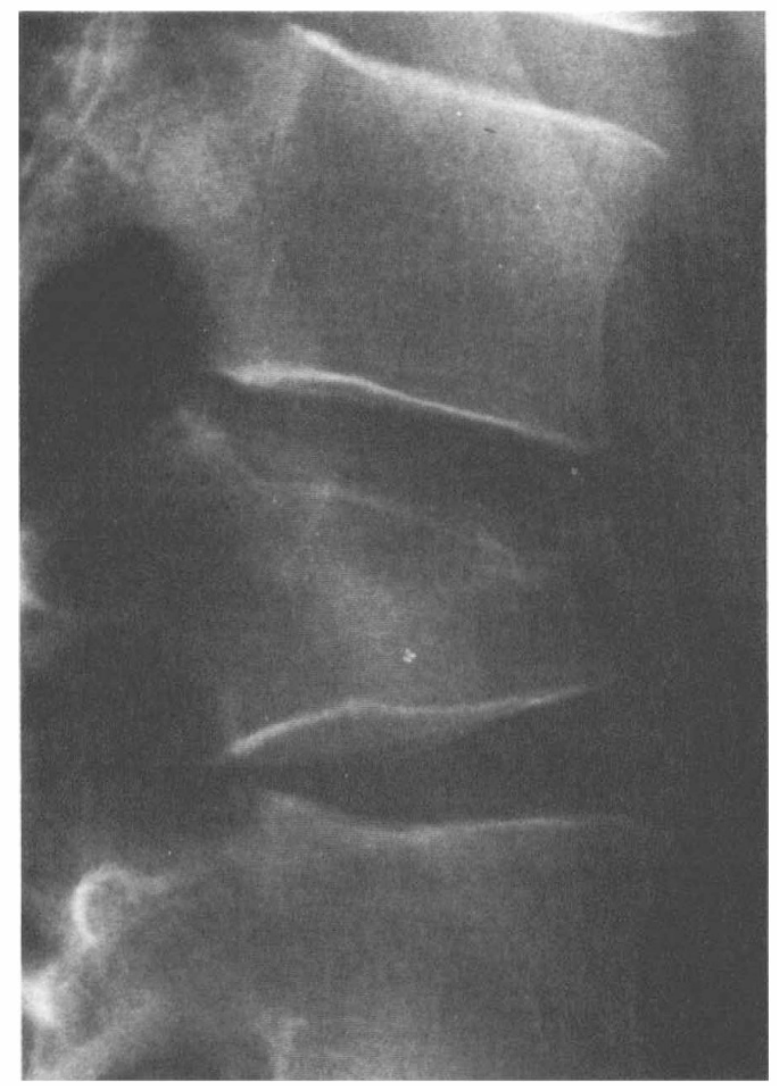

Figure 1 Lateral radiograph of the lumbar spine demonstrating a burst injury of the first lumbar vertebra with some encroachment upon the saggital diameter of the spinal canal.

conus injuries with particular reference to bladder, bowel and sexual functions. This subject has attracted surprisingly little attention in the literature.

\section{Clinical material}

Between 1964 and 1982, 1205 patients with acute spinal cord injury were admitted to the Spinal Cord Injuries Unit at the Royal North Shore Hospital, Sydney. The hospital serves as the major referral centre in New South Wales (population 1981, 5.29 million) for these injuries, and for scattered areas in adjoining states plus sections of the south west Pacific Basin. Twenty one $(1.5 \%)$ of these patients had conus injuries. Because of the referral pattern, 3 patients were lost to followup. Fourteen patients were called for review and 4 others answered detailed questionnaires and were spoken to by telephone. The average follow-up was 4 years, with a range of 18 months to 15 years. There were 16 males and 2 females in the series. The average age at injury was 30.5 years (range 18-30 years).

A fall from a height onto the buttocks, with acute axial loading, was the cause of fracture in 11 patients. Five others sustained injuries in motor vehicle accidents and 2 in industrial accidents. All patients incurred burst fractures in the upper lumbar spine; L1 in 16 patients (Fig. 1) and 2 at L2. 


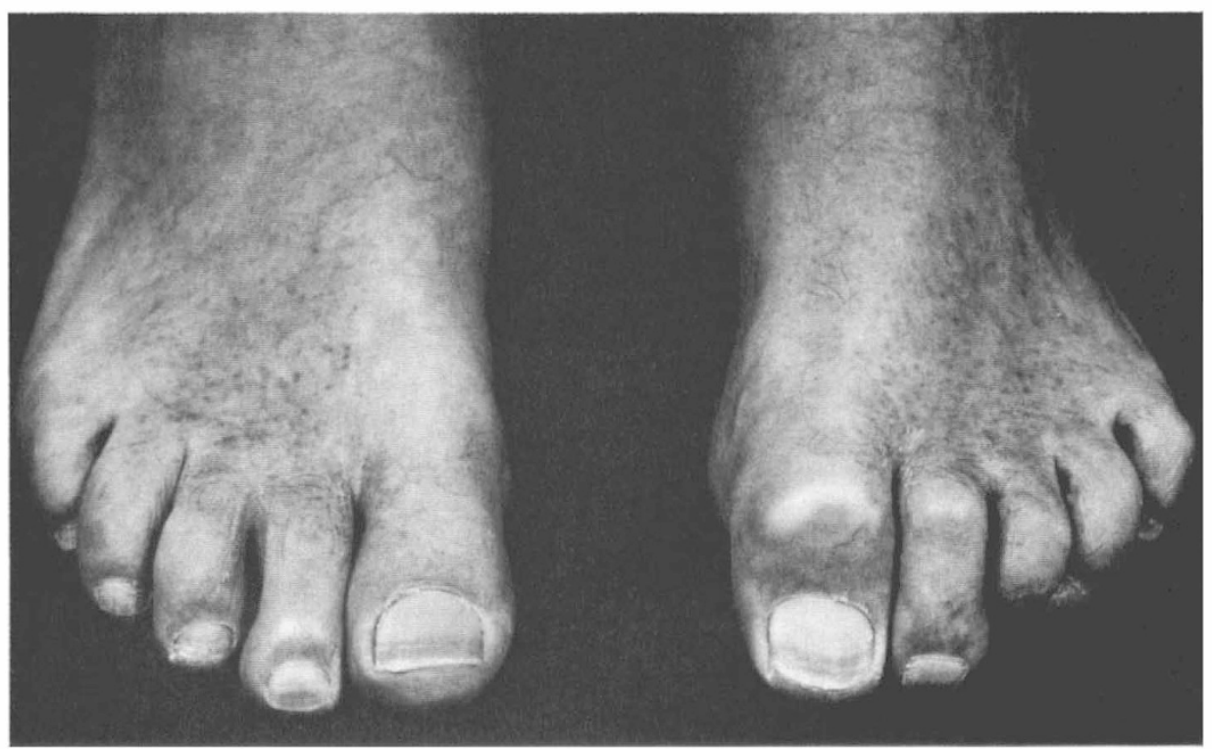

Figure 2 Photographs of the both feet of a patient with a conus injury. Moderate clawing of all toes is present on the left. The motor deficit was limited to the left side, the right being normal.

Because of the time span covered by this study there was no standard protocol of surgical management. Twelve patients were managed operatively, 6 with Harrington rods and local spinal fusion, 4 with Meurig-Williams plates and 2 underwent excision of bony fragments from the spinal canal and anterior interbody fusion without internal fixation. Six patients were managed nonoperatively, and were kept supine for $6-8$ weeks. Then as pain permitted, they were mobilised. Although some patients did improve neurologically from the time of admission, it is impossible to state whether or not spinal realignment with a variable degree of reduction was, in itself, a major factor in the recovery which occurred. Only in 1 patient, where anterior decompression was performed several months after injury, was it clear that the operation had a beneficial effect on bladder function.

\section{Results}

\section{Motor function}

At follow-up, the patients in this series showed little motor deficit and this attests to the relative sparing of the cauda equina. All could walk on discharge from hospital although 4 required some external support. The gait was frequently broad based, without push-off and with abnormal rhythm. At review, 12 patients reported that they were engaged in vigorous sport, 17 drove cars and 12 were employed. One patient was wheelchair-bound for other reasons. A variable degree of calf wasting was evident in 11 patients. In most patients the motor impairment was asymmetrical.

Clawing of the toes was present at follow-up in 9 patients (Fig. 2). The toe deformities tended to become rigid with time but no patient required a surgical 
procedure and they had managed well with minor modifications of footwear. Clawing of the toes is analogous to the intrinsic minus hand.

While there is no clear consensus in the anatomical literature on the segmental value of the intrinsic muscles of the foot, it seems probable that they receive their supply primarily from the first and second sacral segments. For a claw deformity to develop, the long flexors and extensors must be intact, with imbalance expressing itself by hyperextension at the metatarso-phalangeal joints and flexion of the interphalangeal articulations. Support for this concept of pathomechanics comes from the observation that in 9 patients without clawing, 3 had more extensive neurological deficits and in the other 6 , sensation and motor functions were preserved in the first sacral segment to a variable degree.

\section{Sensation}

All patients exhibited a variable degree of sensory loss in the saddle area. In 8 of them the loss was clearly asymmetrical. The cutaneous sensory deficits were associated with few problems. One patient developed trophic ulcers in the perineum from an ill fitting long-leg caliper, and another had suffered a third degree burn from a hot water bottle. While the sensory loss was of little significance to the overall prognosis, there was good correlation of sparing with bladder, bowel and sexual functions.

\section{Bladder function}

Transection of the spinal cord above the level of the conus results in an upper motor neurone bladder where the bladder acts automatically, independent of influence from higher centres. It empties when it is sufficiently distended to excite the reflex arc between the conus and the bladder wall. When the injury is either at the level of the conus or is more distal this reflex arc is interrupted and a flaccid, lower motor neurone bladder results.

The atonic, flabby bladder is progressively distended until either overflow incontinence occurs or it is emptied by abdominal compression. All patients in this series had a lower motor neurone bladder and needed to strain or compress their bladder to micturate. This was usually done on a regular basis every 2-3 hours during the day, with fluid being restricted at night to avoid nocturnal incontinence and overdistention.

Only 1 patient regarded bladder control as completely normal. Eight patients had accepted occasional incontinence which usually occurred with neglect of regular bladder habit, but in 7 of them, intermittent stress incontinence took place. Altered sensation of bladder fullness was present in 12 patients none of whom could interpret the subjective sensation reliably without episodic incontinence. The remainder had no bladder sensation whatsoever. Whilst incontinence is but one aspect of urinary function, it is of major significance to these patients, since dependance on a urine collecting device is a constant remainder of their disability. Nine patients did not wear a urinal, 3 did so at night, and 6 wore a urinal (pad or uridome) at all times.

Urinary infection was a recurrent problem only in 1 patient who had bilateral duplex ureters. The remaining 17 patients, representing 54 patient years, had 
had 28 urinary tract infections in the time span of the study. Five of these were linked to catheterisation during hospitalisation.

In spite of a lower motor neurone bladder being present in all patients, urinary problems were decidedly uncommon. Regular intravenous pyelograms had been performed on all patients and 2 had developed ureteric reflux, and 1 of these was the patient with a duplex collecting system. Four patients had undergone urological procedures, 1 for lithopraxy, 2 for sphincterotomy and 1, a bladder neck resection. These 3 latter patients had all shown high residue volumes after voiding and the surgery was designed to lower this and to avoid infections. No patient had developed clinical, radiological or biochemical evidence of chronic renal insufficiency.

\section{Bowel function}

The level of bowel control differed from that found in patients with higher spinal cord injuries, and is somewhat less efficient. This was assessed by scoring the episodes of incontinence (or accidents) over a period of time. Most patients achieved control by regular daily or second daily bowel actions, with enemas if required, to maintain an empty rectum in between times. Fourteen patients considered that the level of control was satisfactory. Six of them regarded themselves as normal, while 8 lacked control with diarrhoea. Occasional incontinence was seen in 2 patients (approximately once per month) and 2 patients were regularly incontinent. One of these patients stated that most of his bowel actions were accidents. Both patients had generally performed poorly in other aspects of their rehabilitation and the lack of bowel control reflected the patients' inattention to medical advice and diet.

In contrast to the bladder, preservation of rectal and anal sensation was useful in achieving continence. Of the 14 patients with a satisfactory level of control, all had partial preservation of either rectal or perianal sensation, while 4 patients with occasional incontinence had both perianal and rectal anaesthesia. Preservation of sensation is vital in distinguishing flatus from faeces. Involuntary passage of flatus was a distressing symptom for most patients. This was curtailed, in part, by meticulus attention to diet especially avoidance of legumes and to bowel habit.

\section{Sexual function}

Five males stated that they had regained normal potency and were able to have satisfactory sexual intercourse. Two of them had fathered children. Four of them had some sparing of genital sensation. Eight others reported impaired potency with weak or poorly sustained erections. Six of them had intercourse regularly and 1 may have initiated a pregnancy. Two of these men $\left(25^{\circ} \bullet\right.$ ) had some sparing of genital sensation. Three males stated they were impotent but 1 had had limited sexual intercourse albeit without initiating pregnancy. None of these 3 patients had any sparing of genital sensation.

The stimulus to achieve erection varied and was linked with genital sensation. Four of the 5 males who had regained full potency were able to achieve psychogenic and reflex (manual manipulation) erection. Of the other 8 patients 
with impaired potency, 4 could only achieve psychogenic erections and in 1 it was a reflex activity. None of these 5 men had any genital sensation. The 3 other patients in this group achieved impaired erections both by reflex means and psychogenic stimulation. Two of them had retained some genital sensation. None of the totally impotent males had any preservation of penile feeling.

There appears to be a reasonably close correlation between reported potency and genital sensation. On the other hand, libido was unchanged in 15 patients, reported as slightly decreased in 2 others and markedly so in 1 .

Of the 2 women one was post-menopausal and sexually inactive prior to injury. The other had patchy sparing of genital sensation, had been able to engage in satisfactory coitus, but had not attempted conception.

It must be appreciated that assessment of sexual function is far from easy, there being many hidden foibles and no truly objective criteria. Nevertheless, there appears to be a pattern of sexual activity (as detailed above) which relates to neurological function and is paralleled by other findings, notably, genital sensation. The devastating effects of bowel and bladder dysfunction, other functional losses plus the initial impact on ego and self-image doubtless play an important role in the slow return to normal sexual activity after injury. Most men related that it took almost 3 years for return of maximal potency to plateau. One patient was totally impotent for 6 months after injury and then had a gradual return to normality and has since fathered 2 children.

Seven males were of the opinion that the volume of ejaculate had decreased following injury, and several patients were aware of retrograde ejaculation, and noted the subsequent passage of opalescent urine. Several men were of the opinion that the force of ejaculation had decreased after injury thus reflecting the decreased power of the pelvic floor musculature which modulates the act.

\section{Proctalgia and painful dysaesthesia in the lower limbs}

Painful dysaesthesia is a not infrequent concomitant of cauda equina injuries. Proctalgia may be a feature of injuries to the conus, and is sometimes a presenting feature of tumors located in the terminal part of the spinal cord. The combination of proctalgia and distressing dysaesthesae was evident in 4 patients, and in 2 of them it was accompanied by cutaneous hypersensitivity in the L5/S1 dermatomes. In these 2 patients the dysaesthetic state was the major complaint. It was unresponsive to a wide variety of techniques designed to modulate the sensory input (transcutaneous electrical stimulation, sympathetic blocks, etc.).

\section{Low back pain}

It was of some interest to note that in only 1 patient was low back pain a symptom of any severity. This is in general keeping with the long term prognosis of upper lumbar spinal injuries without neurological complications (Agleitti et al., 1983).

\section{Discussion}

By accepted criteria patients with traumatic conus lesions have a relatively good prognosis. Motor function tends to be near normal. Sensory loss, despite being 
in a small but vital area, pales into insignificance when compared with the sensory deprivation associated with more proximal cord injuries. Compared to the latter, in one sense conus patients are a little worse off in that they have major problems with bowel continence, yet have none of the more obvious disabilities of the quadriplegic and paraplegic patient which engender sympathy and form the basis for substantial insurance claims.

By and large, patients with conus medullaris injuries are able to walk well, hold down jobs and play sport. Unlike the patients with high cord injuries they have a low incidence of urological and skin problems. It would appear, other things being equal, that these patients will have a near normal life expectancy.

The reported information on sexual function was somewhat surprising. Two men who remain impotent seem to have accepted their disability without undue complaint or apparent deep concern. One other impotent young male has expressed suicidal intentions because of his loss of virility. The natural history of recovery of potency in this series of patients is a strong indicator that a final assessment of sexual function, often a crucial factor in insurance settlements, should be deferred for at least 3 years post-injury. The technique of nocturnal penile tumescence monitoring has been used but once in the assessment of potential sexual function. In this instance it was negative, and supported the unfavourable prognosis given on the basis of absence of genital sensation. The method appears to hold promise for early evaluation in patients with these injuries as does the intracorporeal injection of either papaverine or phentolamine (Malloy and Malkowiez, 1987). The long term use of these agents to allow neurologically impotent males to engage in sexual intercourse has not yet been reported. The erectile response to direct injection is enhanced after denervation and generally is more positive than when impotence is of vascular origin (Mallory and Malkowiez, 1987).

The conus medullaris occupies but a small fraction of the cross sectional area of the thecal sac at L1. It is therefore not surprising that incomplete lesions occur with those burst fractures which encroach upon canal diameters. Rather, it is surprising that complete conus lesions take place with considerable root escape and this is often asymmetrical. All patients in the present series had, by definition, incomplete lesions. The long term results, strongly suggest that there is a degree of topographical representation within the conus but this has yet to be described. For example, the cell bodies which serve genital sensation are probably in the immediate vicinity of those which relate to potency. Patients with preservation of some anal or rectal sensation achieved better bowel control than those with anal and rectal anaesthesia; both are required for normal control as they act in unison.

As might be expected, the pattern of urinary tract dysfunction observed at follow-up in the present series is closely similar to that reported by Scott (1964) for bladder paralysis secondary to disc prolapse in the distal lumbar spine. This author also recorded remarkably little in the way of long term complications despite extensive bladder denervation. A cystometrogram should be performed on all patients with conus injuries. A flat tracing is the uniform finding but we have recently had under our care 1 patient with a traumatic conus lesion (not included in the present series) in whom an upper motor neurone bladder was demonstrated by cystometrogram in the absence of sensory or motor deficit in 
the lower limbs. In this instance, clearly there are long term implications for bladder management.

A pattern of preservation of sexual function was seen in those patients having some retention of genital sensation, while reduced dependency on urine collecting devices was seen in patients having some perineal sensation. The inter-relationship between cutaneous sensation and long term sexual function is of practical value in the early formulation of prognosis, since the former is assessable soon after injury. Sphincter and sexual function take some months to be fully declared. The presence of penile sensation permits early but cautious optimism which is very reassuring to a man who may otherwise be unable to cope and who is overwhelmed by the apparent extent of his injury.

\section{Acknowledgements}

The authors thank Mrs J. M. Mitchell and Mrs R. Hetherington for their assistance in the preparation of this paper. We are grateful to our colleagues in the Spinal Injuries Unit for permission to review patients under their care.

\section{References}

Malloy TR, Malkowiez B 1987 Pharmacologic treatment of impotence. Urological Clinics of North America 14:297-305.

Scott PJ 1965 Bladder paralysis in Cauda Equina. Fournal of Bone and foint Surgery 47-B:224235.

Aglietti P, Di Muria GV, TAylor TKF, et al. 1983 Conservative treatment of thoracic and lumbar vertebral fractures. Italian fournal of Orthopaedics and Traumatology (English Supplement) 9:83-105. 